\title{
Composición aminoacídica del mosto de los cv. Cigüente, Macabeo, Moscatel de Alejandría y Verdejo cultivados en diversas regiones de España. Efecto del régimen hídrico a lo largo de tres campañas
}

\author{
M.E. Valdés ${ }^{1 *}$, D. Moreno ${ }^{1}$, D. Uriarte ${ }^{1}$, A. Montoro ${ }^{2}$, J.R. Castel ${ }^{\dagger 3}$ y J. Yuste ${ }^{4}$ \\ ${ }^{1}$ Instituto Tecnológico Agroalimentario de Extremadura (CICYTEX), 06071 Badajoz (Spain) \\ ${ }^{2}$ Instituto Técnico Agronómico Provincial, 02007 Albacete (Spain) \\ ${ }^{3}$ Instituto Valenciano de Investigaciones Agrarias, Moncada 46113 Valencia (Spain) \\ ${ }^{4}$ Instituto Tecnológico Agrario de Castilla y León, 47071 Valladolid (Spain)
}

\begin{abstract}
Resumen. Este trabajo analiza el efecto del riego sobre la composición aminoacídica del mosto de 4 variedades blancas cultivadas en diferentes DD.OO. españolas durante el periodo 2012-2014. Se estudió la concentración de aminoácidos precursores de aromas de fermentación: Aspártico (Asp), Isoleucina (Ile), Leucina (Leu), Treonina (Thr), Tirosina (Tyr) y Valina (Val), y la suma de ellos (APN), del aminoácido azufrado Metionina (Met) y del total de aminoácidos libres (FAN). Las variedades, su localización y los tratamientos aplicados fueron: Cigüente (Ci) en Badajoz, secano y riego al 100\% de ETc; Macabeo (Ma) en Albacete, riego a 25\% y 33\% aprox. de ETc; Moscatel de Alejandría (Mo) en Valencia, riego a 50\% y 100\% de ETc; y Verdejo (Ve) en Valladolid, secano y riego a 100\% aprox. de ETc. El mosto analizado tenía un valor de 22-24 ${ }^{\circ}$ Brix. Los análisis de aminoácidos se efectuaron mediante HPLC. Los tratamientos hídricos aplicados modificaron los valores de los aminoácidos en tendencia, significación y extensión según la variedad. En Mo apenas variaron. En Ci y, sobre todo, en Ma el efecto en amplitud y significación dependió del año. En Ve el riego provocó todos los años del estudio idéntica tendencia al descenso en los valores de los aminoácidos. Por tanto, la respuesta de la composición nitrogenada del mosto al régimen hídrico y las condiciones meteorológicas anuales fue diferente en cada variedad cultivada en diferente lugar.
\end{abstract}

\section{Introducción y objetivos}

El viñedo español está mayoritariamente localizado en zonas de clima árido y semiárido. Este hecho, unido a la situación climatológica actual, hace que el riego sea una práctica vitícola cada vez más usual. La cantidad de agua aplicada y el momento de aplicación determinan la composición físico química de la uva $\mathrm{y}$, por tanto, las características del vino elaborado [1] [2]. Además, su efecto también depende en gran medida de la interacción de esta técnica con otros factores, entre los que destacan el cultivar y la situación edafoclimática en la que se encuentre el viñedo, así como el nivel de carga y el equilibrio vegetativo-productivo de las vides, esto es, del denominado efecto "terroir" [3] [4] [5]. Existe una amplia bibliografía en relación con el efecto del estado hídrico de la cepa en variedades tintas [6] [7]. Sin embargo, la información disponible sobre el impacto del riego en variedades blancas es aún escasa. Debido al creciente consumo de vino blanco, el riego se está comenzando a implantar en variedades blancas en la mayoría de zonas vitícolas españolas, siendo necesario el estudio de sus efectos en las variedades típicas y más extendidas en cada zona.

En cuanto a los metabolitos primarios del mosto, la cantidad y la distribución de aminoácidos tienen una importancia primordial. En lo referente a la cantidad global, porque es necesario un nivel mínimo del conjunto de todos ellos, excepto de la prolina (Pro), para el desarrollo y la finalización completa de la fermentación alcohólica. En cuanto a la distribución, porque determinados aminoácidos son precursores de sustancias volátiles, alcoholes, esteres y aldehídos, que se forman durante dicho proceso y que son responsables del aroma del vino y, por tanto, de su calidad organoléptica [8]. Frente a éstos, los aminoácidos azufrados, cistina, cisteína y metionina, son precursores de sustancias que causan mal olor y, por tanto, devalúan la calidad sensorial [9]. La distribución y la cantidad de aminoácidos de un mosto varía en función del cultivar, las condiciones edafoclimaticas, las técnicas de cultivo e incluso con el estado de maduración de la baya [10] [11]. En vista de todo lo anterior, es imprescindible determinar la respuesta, en cuanto a composición aminoacídica de las diferentes variedades en sus diferentes terroirs y conocer el efecto que las prácticas agronómicas tienen en dicha composición.

El objetivo de este trabajo es doble: analizar la composición aminoacídica del mosto procedente de variedades blancas cultivadas en diferentes zonas vitícolas españolas e investigar la variación de dicha composición en función del régimen hídrico y de la campaña. El estudio se ha efectuado en mostos que presentan similar concentración de azúcares, pues se trata de observar la incidencia del estado hídrico en la composición amínica de mostos que producirían vinos de similar grado alcohólico. 


\section{Materiales y métodos}

\subsection{Características de los viñedos}

El experimento se realizó durante las campañas 2012 a 2014 en tres zonas vitícolas de España, con las siguientes variedades: "Cigüente" $(\mathrm{Ci}$, sinónimo Doña Blanca, en Lobón, Badajoz), "Moscatel de Alejandría" (Mo, en Vilamarxant, Valencia), y Verdejo (Ve, en Medina del Campo, Valladolid) y en 2013-2014 con la variedad "Macabeo" (Ma en Albacete). En la tabla 1 se muestran las principales características edafoclimatológicas de los viñedos y los respectivos climas vitícolas [12].

En todos los casos, se trata de viñedos conducidos en espaldera, mediante posicionamiento vertical de la vegetación, podados en cordon Royat biltaeral y sometidos a similares prácticas de cultivo. Los suelos, en todos los casos, son de tipo calcáreo, de reacción alcalina y de mediana a alta capacidad de almacenamiento de agua en general.

Tabla 1. Características de los viñedos en las diferentes localizaciones.

\begin{tabular}{|c|c|c|c|c|}
\hline & Cigüente & Macabeo & Moscatel & Verdejo \\
\hline Coordenadas & $\begin{array}{c}38^{\circ} 03^{\prime} \mathrm{N}, \\
2^{\circ} 06^{\prime} \mathrm{W}\end{array}$ & $\begin{array}{c}39^{\circ} 14^{\prime} \mathrm{N}, 2^{\circ} 5^{\prime} \\
\mathrm{W},\end{array}$ & $\begin{array}{c}39^{\circ} 33^{\prime} \mathrm{N}, \\
0^{\circ} 42^{\prime} \mathrm{W}\end{array}$ & $\begin{array}{c}42^{\circ} 21^{\prime} \mathrm{N}, \\
4^{\circ} 56^{\prime} \mathrm{W}\end{array}$ \\
\hline Altitud, $m$ & 200 & $695 \mathrm{~m}$ & 197 & 785 \\
\hline Patrón & $110 \mathrm{R}$ & $110 R$ & $161-49$ & $110 \mathrm{R}$ \\
\hline Año de plantación & 2005 & 1999 & 1996 & 2006 \\
\hline Marco, $\mathrm{m}$ & $2,95 \times 1,35$ & $3,00 \times 1,5$ & $2,75 \times 1,8$ & $2,6 \times 1,25$ \\
\hline $\mathrm{N}^{0}$ de cepas/repetición & 20 & 18 & 24 & 20 \\
\hline $\mathrm{N}^{0}$ de repeticiones & 4 & 3 & 4 & 4 \\
\hline Textura del suelo & $\begin{array}{c}\text { Franco } \\
\text { Arcilloso }\end{array}$ & $\begin{array}{l}\text { Franco- } \\
\text { Arcilloso- } \\
\text { Arenoso }\end{array}$ & $\begin{array}{l}\text { Franco } \\
\text { Arcilloso }\end{array}$ & $\begin{array}{c}\text { Franco-Arcillo } \\
\text { Arenoso }\end{array}$ \\
\hline Profundidad del suelo, $m$ & 0,75 & $0,5-1,0$ & $>1,5$ & $>1$ \\
\hline Capacidad de retención de agua, $\mathrm{mm} / \mathrm{m}$ & 130 & 130 & 200 & 120 \\
\hline \multirow[t]{2}{*}{ Clima viticola } & $\begin{array}{c}\text { Cálido, } \\
\text { noches frías }\end{array}$ & $\begin{array}{c}\text { Muy cálido, } \\
\text { noches muy } \\
\text { frias }\end{array}$ & $\begin{array}{c}\text { Cálido, } \\
\text { noches } \\
\text { templadas }\end{array}$ & $\begin{array}{c}\text { Cálido, } \\
\text { noches muy } \\
\text { frías }\end{array}$ \\
\hline & Muy seco & Mod. Seco & Mod. seco & Mod. Seco \\
\hline
\end{tabular}

\subsection{Tratamientos de riego y diseños experimentales}

En la Tabla 2 aparecen descritos los tratamientos ensayados en las diferentes localizaciones. Las variables climáticas se midieron en estaciones meteorológicas automáticas ubicadas en cada parcela experimental o en su vecindad, y con ellas se calculó la evapotranspiración de referencia (ETo) mediante la ecuación de PenmanMonteith, [13]. La evapotranspiración del cultivo (ETc) se estimó como producto de ETo y del coeficiente de cultivo (Kc). El riego se aplicó a lo largo del ciclo vegetativo en función de la ETc calculada.

El riego se aplicó por goteo con 1-2 emisores autocompensados por cepa. No hubo problemas de salinidad, dadas las características del suelo y la calidad del agua, que era de relativamente bajo contenido en sal en todos los sitios. El estado hídrico de las plantas se evaluó mediante la medida del potencial hídrico de tallo $(\Psi)$ con cámara de presión [14] [15]. Las mediciones se realizaron a mediodía (11:30 a 12:30 horas solares) a intervalos semanales o quincenales entre Mayo y Octubre, sobre 12 hojas por planta en al menos 6 plantas por tratamiento.
Tabla 2. Descripción de los tratamientos aplicados.

\begin{tabular}{|c||c||c|}
\hline Variedad & Tratamiento & Descripción \\
\hline \hline \multirow{2}{*}{ Cigüente } & Ci-S & No regado \\
\cline { 2 - 3 } & Ci-100R & Riego al 100\% de la ETc potencial \\
\hline \hline \multirow{2}{*}{ Macabeo } & Ma-25R & Riego al 25\% de la ETc potencial \\
\cline { 2 - 3 } & Ma-33R & Riego al 33\% de la ETc potencial \\
\hline \hline \multirow{2}{*}{ Moscatel } & Mo-50R & Riego al 50\% de la ETc potencial \\
\cline { 2 - 3 } & Mo-100R & Riego al 100\% de la Etc potencial \\
\hline \hline \multirow{2}{*}{ Verdejo } & Ve-S & No regado \\
\cline { 2 - 3 } & Ve-100R & Riego al 100\% de la Etc potencial \\
\hline
\end{tabular}

\subsection{Metodología analítica}

El contenido en sólidos solubles se determinó por refractometría (refractómetro digital ATR ST plus Schmidt+Haznsch, Berlin, Germany). El mosto de todos los tratamientos de cada variedad presentó, en el momento del análisis, un valor de Sólidos Solubles Totales de $22 \pm 2{ }^{\circ} \mathrm{Brix}$. El análisis de la composición aminoacídica de la uva de todas las variedades se llevó a cabo en el INTAEX según Valdés et al. [16]. Se efectuó mediante HPLC en un autoanalizador de aminoácidos (Biochrom 30, Pharmacia) empleando una columna intercambiadora de cationes. Como eluyentes se emplearon tampones de citrato de litio de diferente $\mathrm{pH}$. Una vez separados, los aminoácidos reaccionan con ninhidrina y se detectan por espectrofotometría a $570 \mathrm{~nm}$, excepto la prolina (Pro), que se detecta a $440 \mathrm{~nm}$. La cuantificación se efectuó mediante calibración externa, empleando una recta de calibrado para cada aminoácido. A partir de las concentraciones de todos los aminoácidos se evaluó el valor de la concentración total de aminoácidos libres (Free Aminoacids Nitrogen, FAN) y de aminoácidos asimilables por las levaduras (Aminoacid Assimilable Nitrogen, AAA-N), restándole a FAN el valor de Pro. El cálculo de la concentración total de aminoácidos precursores de aromas de fermentación (Precursors Aminoacid Nitrogen) se efectuó como sumatorio de los aminoácidos Aspártico (Asp), Isoleucina (Ile), Leucina (Leu), Fenilalanina (Phe), Treonina (Thr), Tirosina (Tyr) y Valina (Val).

\section{Análisis estadístico}

En cada variedad, para determinar el efecto del régimen hídrico cada año, los datos se sometieron a un test de Student. Para establecer la significación del efecto año, en cada variedad y régimen hídrico se efectuó un ANOVA y el posterior test de separación de medias. La significación del factor variedad, se determinó mediante un ANOVA y el posterior test de medias al que se sometieron los datos procedentes de todos los tratamientos y años de todas las variedades. Con los datos medios procedentes de todos los tratamientos y años se realizó un ANOVA y el posterior test de medias y un análisis de componentes principales (ACP). El análisis estadístico fue ejecutado mediante el software informático XLstat-Pro (Addinsoft, París 2011). 


\section{RESULTADOS Y DISCUSIÓN}

\subsection{Estado hídrico de las cepas}

La tabla 3 muestra las lluvias registradas durante los años del estudio y el estado hídrico de las cepas, a través del potencial hídrico de tallo de los diferentes tratamientos en las diferentes localizaciones.

Los datos expuestos en la tabla 3 muestran que la campaña 2013 fue la más lluviosa en todas las zonas vitícolas y que la más seca fue la 2012 en $\mathrm{Ci}$ y Ve y la 2014 en Mo. Respecto a Ma, las dos campañas en estudio, (2013 y 2014) fueron climatológicamente muy diferentes, mucho más húmeda la de 2013.

Tabla 3. Precipitaciones anuales, agua de riego aportada y estado hídrico (potencial de tallo) de las cepas durante el período vegetativo de las diferentes campañas

\begin{tabular}{|c|c|c|c|}
\hline \multirow{3}{*}{ Variedad } & \multicolumn{3}{|c|}{ Precipitaciones anuales registradas ( $\mathrm{mm}$ ) } \\
\hline & \multicolumn{3}{|c|}{ Año } \\
\hline & 2012 & 2013 & 2014 \\
\hline Cigüente & 268 & 585 & 514 \\
\hline Macabeo & & 480 & 187 \\
\hline Moscatel & 280 & 352 & 197 \\
\hline Verdejo & 122 & 407 & 329 \\
\hline \multirow{3}{*}{ Tratamiento } & \multicolumn{3}{|c|}{ Agua aportada ( $\mathrm{mm})$} \\
\hline & \multicolumn{3}{|c|}{ Año } \\
\hline & 2012 & 2013 & 2014 \\
\hline Ci-S & 0,00 & 0,00 & 0,00 \\
\hline Ci-100R & 356,46 & 359,77 & 294,27 \\
\hline Ma-25R & & 70,00 & 123,61 \\
\hline Ma-33R & & 84,00 & 151,61 \\
\hline Mo-50R & 156,05 & 120,00 & 131,09 \\
\hline Mo-100R & 280,64 & 233,82 & 261,32 \\
\hline Ve-s & 0,00 & 0,00 & 0,00 \\
\hline Ve-100R & 250,71 & 208,28 & 216,05 \\
\hline \multirow{3}{*}{ Tratamiento } & \multicolumn{3}{|c|}{ Utallo medio periodo vegetativo ( $\mathrm{MPa}$ ) } \\
\hline & \multicolumn{3}{|c|}{ Año } \\
\hline & 2012 & 2013 & 2014 \\
\hline Ci-S & $-1,29$ & $-1,18$ & $-1,09$ \\
\hline Ci-100R & $-0,56$ & $-0,62$ & $-0,65$ \\
\hline Ma-25R & & $-0,88$ & $-1,20$ \\
\hline Ma-33R & & $-0,61$ & $-0,90$ \\
\hline Mo-50R & $-0,81$ & $-0,69$ & $-0,75$ \\
\hline Mo-100R & $-0,78$ & $-0,61$ & $-0,66$ \\
\hline Ve-s & $-1,18$ & $-1,00$ & $-1,07$ \\
\hline Ve-100R & $-0,77$ & $-0,73$ & $-0,81$ \\
\hline
\end{tabular}

En general, el riego disminuyó el estrés hídrico de las cepas de todas las variedades (valores de $\Psi$ menos negativos). La reducción dependió en gran medida de la variedad considerada y para cada una de ellas, de la campaña considerada. Según los datos mostrados en la tabla 3, durante las tres campañas del estudio las cepas Ci-S y las Ve-S mostraron un grado de estrés [17] catalogado como moderado a fuerte, y el de las Ci-100R y Ve-100R fue leve según. La aplicación de riego en Mo fue la que menor efecto provocó en el estado hídrico de las cepas que mantuvieron siempre un grado de estrés clasificado como leve a moderado. Finalmente, en Ma la situación fue muy diferente en cada una de las dos campañas analizadas: en 2013 el grado de estrés de las Ma-25R y las Ma-33R fue moderado a fuerte y leve a moderado respectivamente; sin embargo, en 2014 las cepas Ma-25R sufrieron un estrés severo y las Ma-33R fuerte a severo.

\subsection{Composición aminoacídica del mosto}

\subsubsection{Caracterización varietal}

La tabla 4 muestra los valores medios interanuales de los aminoácidos analizados y del PAN, FAN y AAA-N de los de las variedades en estudio

De entre los aminoácidos precursores aromáticos Thr y $\mathrm{Va}$, fueron en este orden, los que alcanzaron los mayores valores medios interanuales en todas las variedades estudiadas. Frente a ellos, Met fue el de menor concentración en todas ellas.

En todas las variedades, los valores medios de AAA$\mathrm{N}$ se hallan en los intervalos normales para este parámetro. En todos los casos, la concentración es suficiente para asegurar una buena marcha de la fermentación alcohólica y una correcta finalización de la misma. La variedad más rica en aminoácidos fue Mo, cuyos valores medios de FAN fue superior a los del resto de variedades. Los mostos Ci presentaron los valores más bajos de este parámetro debido, entre otras causas, a los altos valores de Prolina, aminoácido no asimilable por las levaduras, hallados en esta variedad (valores no mostrados).

Tabla 4. Composición aminoacídica media interanual (20122014) de las variedades Cigüente, Macabeo, Moscatel y Verdejo.

\begin{tabular}{|c|c|c|c|c|}
\hline Aminoácidos (mg/L) & | Cigüente & Macabeo & || Moscatel & Verdejo \\
\hline Asp & $20,04 a^{1}$ & $18,67 a b$ & $14,67 \mathrm{~b}$ & $24,68 a$ \\
\hline Ile & $11,56 b$ & $8,59 \mathrm{~b}$ & $11,86 \mathrm{ab}$ & $15,17 a$ \\
\hline Leu & $22,87 a$ & $14,65 b$ & $25,19 a$ & $20,68 a$ \\
\hline Phe & $19,23 b c$ & $17,46 \mathrm{c}$ & $23,73 a b$ & $25,12 a$ \\
\hline Thr & $53,33 b$ & $50,97 \mathrm{~b}$ & $39,71 \mathrm{c}$ & $87,39 a$ \\
\hline Tyr & $7,34 \mathrm{c}$ & $19,04 a$ & $16,35 \mathrm{ab}$ & $10,68 \mathrm{bc}$ \\
\hline Val & $33,01 a$ & $29,88 a$ & $28,43 a$ & $32,59 a$ \\
\hline Met & $4,65 a$ & $1,59 \mathrm{~b}$ & $4,41 \mathrm{a}$ & $4,28 \mathrm{a}$ \\
\hline \multicolumn{5}{|c|}{ Parámetros aminoacídicos (mg/L) } \\
\hline PAN & $167,39 b$ & $159,27 b$ & $159,94 b$ & $216,32 a$ \\
\hline FAN & $1366,22 b$ & $1638,91 \mathrm{a}$ & $1756,67 a$ & $1373,47 b$ \\
\hline AAA-N & $983,03 b$ & $1029,59 b$ & $1263,91 a$ & $1094,94 b$ \\
\hline
\end{tabular}

${ }^{1}$ Para un mismo aminoácido o parámetro, letras diferentes indican diferencias entre variedades $(p<0,05)$

En Ve se registraron los mayores valores medios interanuales de Asp, Ile, Phe y especialmente Thr. Estos aminoácidos son importantes precursores de aromas de fermentación [18]. En consecuencia, el valor medio de PAN fue superior a las restantes. El valor medio del PAN 
representa en esta variedad un 16\% del FAN, superior al $12 \%, 10 \%$ y $9 \%$ en Ci, Ma y Mo. De estos resultados aquí expuestos puede deducirse que en función de su composición aminoácidica, Ve es la variedad con el potencial de aromas de fermentación más elevado de entre las analizadas.

\subsubsection{Efecto de la campaña y del régimen} hídrico

La tabla 5 muestra el efecto del año, el régimen hídrico y la interacción de ambos factores en la concentración de aminoácidos y en ambos parámetros analizados. En todas las variedades, y en ambos regímenes hídricos establecidos en cada una de ellas, la mayoría de aminoácidos y parámetros analizados, se vieron afectados de forma significativa por el factor año. Similares resultados hallaron Vilanova et al. en la variedad Mencía cultivada en diferentes zonas de la Ribeira Sacra (España) [19].

En la variedad Cigüente, los mayores valores de sustancias nitrogenadas se observaron en ambos regímenes hídricos, en el año más seco, el 2012, y en consecuencia el valor de FAN de ese año fue significativamente superior al de las otras dos. También en los dos tratamientos aplicados en Mo, las mayores concentraciones de aminoácidos también se registraron en la campaña más seca, que en esa localización fue la 2014. Recíprocamente, en Ve los valores más pequeños correspondieron al 2013 que fue la campaña más húmeda.

La amplitud y la significación del efecto de la campaña efecto fueron superiores a los del régimen hídrico, conclusión a la que también llegaron los estudios de Ortega et al. realizados en la variedad Verdejo en la D.O. Rueda, así como Bouzas et al. en el cultivar Albariño procedentes de Rías Baixas y Ribeiro [20] [21]. Hay que destacar que la variación debida a la campaña fue muy similar en cuanto a significación y extensión en los dos regímenes hídricos impuestos en cada variedad, de manera que, mediante la aplicación de agua en el caso de Ci y Ve o la aplicación de agua en mayor cantidad en los casos de Mo y Ma, no se evitaron las variaciones debidas al efecto campaña. Además, se observó interacción significativa tratamiento*año en un número variable de aminoácidos y parámetros según la variedad: 5 en $\mathrm{Ci}, 2$ en Mo, 2 en Ve y en todos excepto Ile en Ma, variedad en la que el efecto del riego sobre la concentración de las sustancias nitrogenadas varió en función del año considerado.

En ninguna de las campañas en estudio de $\mathrm{Ci}$, la aplicación de riego (Ci-S vs Ci-100R) modificó significativamente la concentración total de aminoácidos libres (FAN). En cambio, sí tuvo efecto sobre la de algunos aminoácidos, en concreto los valores de Phe de $\mathrm{Ci}-100 \mathrm{R}$ son menores que los de $\mathrm{Ci}-\mathrm{S}$ en las tres campañas. Este resultado puede tener una repercusión negativa en el aroma de los vinos ya que este aminoácido es precursor del 2-fenil etanol, sustancia formada durante la fermentación alcohólica que aporta aroma a rosa a los vinos. En la campaña 2014, se observó un descenso generalizado en los valores de los aminoácidos mostrados en la tabla 4, que provocó una disminución del valor del
PAN que del $15 \%$ en Ci-S pasó a suponer únicamente un $10 \%$ en $\mathrm{Ci}-100 \mathrm{R}$. Únicamente en esta campaña, a diferencia de las otras dos anteriores, el valor de Asp en $\mathrm{Ci}-100 \mathrm{R}$ fue menor $(\mathrm{p}<0,05)$ que en $\mathrm{Ci}-\mathrm{S}$.

La respuesta a la aplicación de una mayor cantidad de agua de riego en la variedad Macabeo (Ma-25R vs Ma33R) sobre la concentración de las sustancias en estudio, fue muy diferente en los dos años analizados. El primer año de la aplicación del riego no se observó ningún cambio significativo, en tanto que, en 2014, año más seco y en el que la aplicación de agua en Ma-33R fue superior al año anterior, se registró un aumento significativo de todos los aminoácidos y en consecuencia de los parámetros nitrogenados.

En la variedad Mo el estado hídrico de la cepa en las campañas 2012 y 2013 no tuvo repercusión en las concentraciones individualizadas de ninguno de los aminoácidos analizados En 2014, en Mo-100R respecto de Mo-50R, excepto en Asp, se hallaron descensos generalizados, de escasa amplitud en todos los casos, en los valores de todos los aminoácidos que fueron significativos en Phe, Thr. Estos descensos provocaron un descenso significativo del $8 \%$ del PAN. También se registraron disminuciones $(\mathrm{p}<0,05)$ del $7 \%$ de AAA-N y del 13\% del FAN.

Finalmente, en la variedad Ve, la aplicación de agua (Ve-S vs Ve-100R) provocó en las tres campañas una similar tendencia al descenso en los valores de prácticamente todos los aminoácidos, excepto de Asp. En el año 2012, se hallaron disminuciones significativas de Ile, Leu, Phe, en 2013 de Ile, Leu, Thr, y Val, y en el 2014 únicamente de Ile. Estos resultados coinciden parcialmente con los de Ortega et al. [19]. En sus investigaciones, al igual que en este trabajo, el riego provocó descensos de los valores de Thr, Sin embargo, a diferencia de los resultados hallados en este trabajo, estos investigadores hallaron que, en uvas maduras, el riego en esta variedad provocó un aumento de la concentración de aminoácidos. Por otra parte, es interesante destacar que el sentido de la variación provocada por el riego dependió en gran medida de la madurez de las uvas. A este respecto hay que señalar que, en este estudio, el análisis se ha efectuado siempre en uvas maduras.

De lo anterior podría deducirse que la respuesta de la composición nitrogenada al estado hídrico de la cepa es diferente para cada cultivar en su medio tradicional. En algunas variedades, la repuesta es independiente de la climatología anual (Mo y Ve), frente a otras en la que respuesta dependió de ese factor ( $\mathrm{Ci}$ y $\mathrm{Ma})$. Centrándonos en estas últimas, en el caso de $\mathrm{Ci}$, la respuesta tuvo siempre igual sentido pero diferente amplitud y en el caso de esta última varió en amplitud y signo. 
Tabla 5. Efecto de la campaña y el tratamiento en la composición aminoacídica del mosto de las variedades Cigüente, Macabeo, Moscatel y Verdejo.

\begin{tabular}{|c|c|c|c|c|c|c|c|c|c|c|c|c|c|}
\hline \multirow{2}{*}{$\begin{array}{c}\text { Aminoácidos } \\
\text { (mg/L) }\end{array}$} & \multirow[b]{2}{*}{ Año } & \multicolumn{3}{|c|}{ Ciguente } & \multicolumn{3}{|c|}{ Macabeo } & \multicolumn{3}{|c|}{ Moscatel } & \multicolumn{3}{|c|}{ Verdejo } \\
\hline & & Cis & Ci100R & Tr*año $^{3}$ & Ma25R & Ma33R & Tr*año & Mo50R & Mo100R & Tr*año & Ves & VeR & |Tr*año \\
\hline \multirow[b]{3}{*}{ Asp } & 2012 & $25,54 a^{1}$ & $40,76 a^{* * *^{2}}$ & \multirow{3}{*}{$* *$} & & & \multirow{3}{*}{$* * *$} & $16,53 a$ & $16,82 a$ & \multirow{3}{*}{ ns } & $28,66 a$ & $39,62 a^{* * *}$ & \multirow{3}{*}{ * } \\
\hline & 2013 & $13,55 b$ & $18,59 b^{*}$ & & 23,00 & 16,23 & & $15,52 a$ & $15,56 a$ & & $12,96 \mathrm{c}$ & $11,00 \mathrm{~b}$ & \\
\hline & 2014 & $14,06 \mathrm{~b}$ & $7,75 c^{\star * \star}$ & & 10,74 & $25,20^{4 \star^{\bullet \cdots}}$ & & $11,37 b$ & $12,24 b$ & & $22,71 \mathrm{~b}$ & $33,15 a^{*}$ & \\
\hline \multirow[b]{3}{*}{ lle } & 2012 & 16,93 & $11,46 a$ & \multirow{3}{*}{ ns } & & & \multirow{3}{*}{ * } & $8,06 \mathrm{c}$ & $8,84 b$ & \multirow{3}{*}{ ns } & 29,75 & $9,73^{*}$ & \multirow{3}{*}{ ns } \\
\hline & 2013 & 10,88 & $10,27 a$ & & 9,34 & 10,40 & & $12,91 b$ & $12,28 a$ & & 14,31 & $8,79^{* * *}$ & \\
\hline & 2014 & 14,02 & $5,83 b^{*}$ & & 5,80 & $9,46^{*^{\bullet}}$ & & $15,20 a$ & $13,85 a$ & & 19,98 & $8,48^{*}$ & \\
\hline \multirow[b]{3}{*}{ Leu } & 2012 & 29,31 & $30,88 a$ & \multirow{3}{*}{ ns } & & & \multirow{3}{*}{$* * *$} & $17,50 c$ & $19,94 b$ & \multirow{3}{*}{ ns } & $38,36 a$ & $16,83 a^{*}$ & \multirow{3}{*}{ ns } \\
\hline & 2013 & 20,79 & $20,16 b$ & & 16,25 & 16,94 & & $27,32 b$ & $26,28 a$ & & $19,36 b$ & $10,99 b^{* * *}$ & \\
\hline & 2014 & 21,78 & $14,31 c^{*}$ & & 9,20 & $17,19^{*^{\bullet \bullet \bullet}}$ & & $31,21 a$ & $28,85 a$ & & $24,63 a b$ & $13,92 \mathrm{ab}$ & \\
\hline \multirow[b]{3}{*}{ Phe } & 2012 & $30,32 a$ & $21,66 a^{*}$ & \multirow{3}{*}{ * } & & & \multirow{3}{*}{$* * *$} & $17,11 \mathrm{c}$ & $18,32 c$ & & $35,69 a$ & $18,71^{*}$ & \\
\hline & 2013 & $16,26 b$ & $12,99 b^{*}$ & & 25,08 & 22,86 & & $25,18 b$ & $23,38 b$ & ns & $22,67 a$ & 16,72 & ns \\
\hline & 2014 & $26,19 a b$ & $7,96 c^{*}$ & & 6,85 & $18,32^{*^{\bullet \bullet \bullet}}$ & & $30,36 a$ & $28,01 a^{*}$ & & $35,43 a$ & 21,49 & \\
\hline & 2012 & $76,49 a$ & $66,70 a$ & & & & & $33,80 a$ & $35,94 b$ & & $95,78 \mathrm{ab}$ & 95,99 & \\
\hline & 2013 & $34,45 c$ & $43,44 b$ & $* *$ & 46,70 & 34,11 & $* * *$ & $32,27 \mathrm{~b}$ & $30,59 c$ & ns & $78,53 b$ & $52,11^{* * *}$ & ns \\
\hline Thr & 2014 & $58,34 b$ & $40,55 b^{*}$ & & 46,35 & $71,43^{*^{\bullet \bullet \bullet}}$ & & $55,48 a$ & $50,16 a^{*}$ & & $105,88 a$ & 96,03 & \\
\hline & 2012 & $5,38 \mathrm{~b}$ & 5,48 & & & & & $10,72 b$ & $11,31 \mathrm{~b}$ & & $7,47 \mathrm{~b}$ & $7,43 b$ & \\
\hline & 2013 & $4,72 b$ & 5,22 & ns & 4,38 & 4,66 & $* * *$ & $9,11 \mathrm{~b}$ & $7,97 \mathrm{c}$ & * & $8,17 \mathrm{~b}$ & $6,96 \mathrm{~b}$ & * \\
\hline Tyr & 2014 & $11,09 a$ & $12,17^{*}$ & & 13,30 & $46,56^{*^{\bullet}}$ & & $31,77 a$ & $27,19 a$ & & $21,54 a$ & $12,53 a$ & \\
\hline & 2012 & $40,07 a$ & $42,18 a$ & & & & & $22,14 c$ & $23,99 \mathrm{~b}$ & & $48,61 a$ & $29,69 a$ & \\
\hline & 2013 & $27,28 \mathrm{~b}$ & $26,33 b$ & ns & 23,57 & 21,49 & $* * *$ & $25,76 \mathrm{~b}$ & $25,01 b$ & ns & $25,68 \mathrm{~b}$ & $18,05 b^{\star *}$ & ns \\
\hline Val & 2014 & $34,81 \mathrm{ab}$ & $27,41 b^{*}$ & & 27,64 & $43,14^{* \bullet \bullet}$ & & $38,17 a$ & $35,51 a$ & & $44,11 \mathrm{ab}$ & $29,40 a$ & \\
\hline & 2012 & $9,68 a$ & $7,75 a$ & & & & & $4,21 \mathrm{~b}$ & 4,62 & & 7,85 & 4,31 & \\
\hline & 2013 & $2,82 b$ & $4,13 b$ & ns & nd & nd & $* * *$ & 4,49ab & 4,42 & ns & 3,09 & 0,85 & ns \\
\hline Met & 2014 & $2,64 b$ & $0,86 c^{*}$ & & nd & nd & & $4,69 a$ & 4,02 & & 6,63 & 2,94 & \\
\hline & & & & & arámetr & os aminoa & cídicos & & & & & & \\
\hline & 2012 & $224,04 a$ & $219,12 a$ & & & & & $125,95 \mathrm{c}$ & $135,16 \mathrm{~b}$ & & $284,33 a$ & $218,01 a$ & \\
\hline & 2013 & $127,94 b$ & $137,01 b$ & * & 148,32 & 126,68 & $* * *$ & $148,09 b$ & $141,08 b$ & ns & $181,68 b$ & $124,63 b$ & ns \\
\hline PAN & 2014 & $180,29 \mathrm{ab}$ & $115,98 b^{*}$ & & 119,88 & $231,31^{*}$ & & $213,56 a$ & $195,81 a^{*}$ & & $274,28 \mathrm{ab}$ & $215,01 \mathrm{a}$ & \\
\hline & 2012 & $1185,89 a$ & $1291,31 a$ & & & & & $1105,90 \mathrm{~b}$ & $1153,18 b$ & & $1207,54 a b$ & $1062,10 \mathrm{a}$ & \\
\hline & 2013 & $618,07 b$ & $922,05 b^{* * *}$ & $* *$ & 866,55 & 870,00 & $* * *$ & $1093,87 b$ & $1061,84 b$ & ns & $963,34 b$ & $756,29 b$ & ns \\
\hline AAA-N & 2014 & $958,98 a$ & $921,86 b$ & & 971,46 & $1329,69 * 0.0$ & & $1643,14 a$ & $1525,48 a^{*}$ & & $1333,29 a$ & $1247,11 a$ & \\
\hline & 2012 & $1732,62 a$ & $1798,95 a$ & & & & & $1515,97 b$ & $1600,71 b$ & & 1434,48 & $1351,66 a$ & \\
\hline & 2013 & $1147,68 b$ & $1132,35 b$ & ns & 1330,25 & 1127,61 & $* * *$ & $1581,86 b$ & $1488,10 b$ & * & 1330,76 & $910,71 b$ & ns \\
\hline FAN & 2014 & $1208,01 b$ & $1177,73 b$ & & 1616,49 & $2276,29^{* \cdots}$ & & $2330,81 a$ & $2022,57 a^{* *}$ & & 1678,61 & $1534,61 a$ & \\
\hline
\end{tabular}

${ }^{1}$ Diferentes letras para un mismo aminoácido y tratamiento indican diferencias significativas entre distintas campañas $(\mathrm{p}<0,05)$. $2 *, * * y * * *$ indican para un determinado aminoácido y campaña, diferencias significativas $(\mathrm{p}<0,05, \mathrm{p}<0,01$ y $\mathrm{p}<0,001)$ debidas al tratamiento.

$3 *, * * \mathrm{y} * * *$ indican interacción significativa tratamiento $\mathrm{x}$ año $(\mathrm{p}<0,05,0,01$ y 0,001 respectivamente).

$4 \cdot, \cdots$ y $\cdots$ en un mismo aminoácido y tratamiento en la variedad Macabeo, indican diferencias significativas ( $p<0,05, p<0,01$ y $p<0,001)$ entre las dos campañas analizadas. 
Con el objetivo de facilitar la interpretación de los resultados correspondientes a la incidencia de los diferentes tratamientos en la composición aminoacídica interanual de los mostos, se sometieron los valores medios interanuales de cada tratamiento de $\mathrm{Ci}$, Mo y Ve a un análisis de componentes principales. La razón de no incluir Ma se debe a que únicamente se dispone de dos años de esta variedad, y el comportamiento fue de diferente signo en cada uno de ellos. La figura 1 muestra los resultados de la aplicación del Análisis de Componentes Principales (PCA). El componente principal F1, que explica el $45,46 \%$ de la varianza total, permite diferenciar los tratamientos VeS y CiS de Ve100R y Ci100R respectivamente. Los primeros están situados a la derecha del eje de modo que poseen mayores valores de Ile, Met, Phe y PAN. Sin embargo, los valores de estos aminoácidos apenas diferencian Mo-50R de Mo100R.

La componente principal F2, asociada principalmente a los valores de Thr en el eje positivo y a FAN en el negativo, permite diferenciar las muestras procedentes de los tratamientos con mejor estado hídrico procedentes de las diferentes variedades en función de los valores de Thr, Asp, FAN y AAA$\mathrm{N}$, de manera que las muestras con mayores valores de Thr son las Ve-100R, mientras que las de Mo-50R son las de mayor FAN. También este eje permite constatar la poca diferencia exixtente entre la composición de las muestras M-100R y la delas M-50R

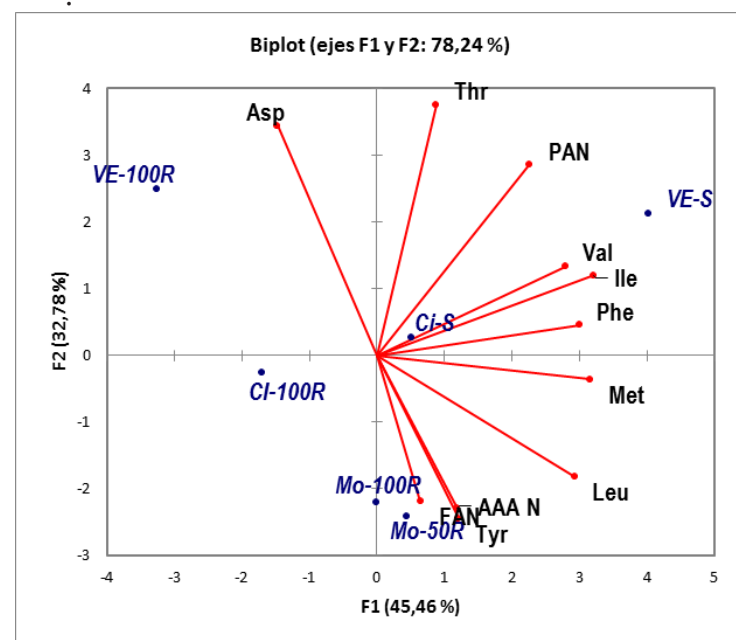

Figura 1. Análisis de Componentes Principales (PCA) de los valores medios interanuales de los aminoácidos de los diferentes tratamientos.

Por tanto, de lo anterior, puede concluirse que no se puede establecer un efecto generalizado del riego sobre la concentración de aminoácidos precursores de aromas de fermentación. Los resultados aquí expuestos indican que la respuesta depende en gran medida del binomio variedad-terroir y dentro de cada uno de ellos, incluso de la campaña en estudio.

Son necesarios más estudios de estas variedades en otras localizaciones para determinar respectivamente el grado de contribución de la variedad y del terroir a la respuesta del viñedo observada.

\section{BIBLIOGRAFÍA}

1. E. Gamero, D. Moreno, M. Vilanova, D. Uriarte, M.H. Prieto, M. E. Valdés, Aust. J. Grape Wine Res. 20(3), 394-400 (2014)

2. I. Talaverano, E. Valdés, D. Moreno, E. Gamero, L. Mancha, M. Vilanova, J. Sci. Food Agric. 97 (5), 1533-1542 (2017)

3. E. Vadour, Les terroirs viticoles. Definitions, characterisation, protection (EDP Dunod, Paris, 2003)

4. M. E. Valdés, M. H. Prieto, D. Moreno, E. Gamero, A. Montoro, F. Mañas, R. López-Urrea, P. LópezFuster, J. Yuste, M.V. Alburquerque, J.R. Yuste, E. Barajas, A. Yeves, D. Pérez, D.S. Intrigliolo, J.R. Castel, IXe International Terroirs Congress (Bourgogne-Dijon, Champagne-Reims, 2012)

5. J.R. Castel, M. E. Valdés, M. H. Prieto, D. Uriarte, L. Mancha, A. Montoro, F. Mañas, R. López-Urrea, P. López-Fuster, J. Yuste, M.V. Alburquerque, J.R. Yuste, E. Barajas, A. Yeves, D. Pérez, D.S. Intrigliolo, IXe International Terroirs Congress (BourgogneDijon, Champagne-Reims, 2012)

6. P. Romero, R. Gil-Muñoz, F. M. Amor, M. E. Valdes, J. I. Fernández, A. Martinez-Cutillas, Agr. Water Manag. 121, 85-101 (2013)

7. I. Garrido, J. Llerena, M. E. Valdés, L. Mancha, D. Uriarte, M. H. Prieto, F. Espinosa, J. Int. Sci. Vigne Vin. 48, 1-32 (2014)

8. P. Hernández-Orte, J. F. Cacho, V. Ferreira,. J. Agric. Food Chem. 50, 2891-2899 (2002)

9. N. Moreira, F. Mendes, O. Pereira, P. Guedes de Pinho, T. Hogg, I. Vasconcelos,.Anal. Chim. Acta. 458,157-167 (2002)

10. M.L Asensio, M.E. Valdes, F. Cabello, Characterisation of some Spanish white grapevine cultivars by morphology and amino acid analysis. Sci. Hortic. 93, 289-299 (2002)

11. T. Garde-Cerdán, C. Lorenzo, J.F. Lara, F. Pardo, C. Ancín-Azpilicueta, M.R. Salinas, J. Agric. Food Chem. 57:2410-2419 (2009)

12. J. Tonietto, A. Cabonneau, Agric. Forest Meteorol. 124, 81-97 (2004)

13. R.G. Allen, W.O. Pruitt, M.E Jensen, Lysimeters for evapotranspiration and environmental measurements. (EDP Allen RG, Howell TA, Pruitt WO, Walter IA, Jensen MEASCE, New York, 1991)

14. X. Chone, C. Van Leeuwen, D. Dubourdieu, J. P. Gaudillère, Ann. Bot. 87, 477-483. (2001)

15. P.F. Scholander, H.T. Hammel, E.D. Bradstreet, E.A. Hemmingsen, Science 148, 339-346 (1965)

16. M. E. Valdés, M. Vilanova, E. Sabio, M. J. Bernalte, Food Chem. 125, 430-437 (2011)

17. A. Deloire, H. Ojeda, O. Zebic, N. Bernard, J.J. Hunter, A. Carbonneau. www.infowine.com, revue internet de viticulture et oenologie 19 (2006)

18. Guitart, A., Hernandez-Orte, P., Ferreira, V., Pena, C., Cacho, J. Am. J. Enology Vitic. 50, 253-258. (1999)

19. M. Vilanova, I. Rodríguez, P. Canosa, I. Otero, E. 
Gamero, D. Moreno, I. Talaverano, M. E. Valdés, Food Chem. 169, 187-196 (2015)

20. M. Ortega-Heras, S. Pérez-Magariño, V. Del-VillarGarrachón, C., González-Huerta, L.C. Moro, A. Guadarrama, S. R., Villanueva Gallo, S. Martín de la Helguera, J. Sci. Food Agric. 94, 2073-2082 (2014).

21. Y. Bouzas-Cid, E. Díaz-Losada, E. Trigo-Córdoba, E. Falqué, I. Orriols, T. Garde-Cerdán, J. M. MirásAvalos, Sci. Hortic, 227, 313-325 (2018) 